\title{
Modelling of a five reactor Activated Sludge cascade process using ASM \#1
}

\author{
S. Watt $^{\mathrm{a}}$ (D) and M. Nelson ${ }^{\mathrm{b}}$ (D) and F. Hai ${ }^{\mathrm{c}}$ (D) and H. Sidhu

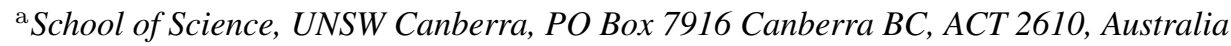 \\ ${ }^{\mathrm{b}}$ School of Mathematics and Applied Statistics, University of Wollongong, NSW 2522, Australia \\ ${ }^{\mathrm{c}}$ Strategic Water Infrastructure Lab, School of Civil, Mining and Environmental Engineering, University of \\ Wollongong, NSW 2522, Australia \\ Email: simon.watt@adfa.edu.au
}

\begin{abstract}
The activated sludge process is the most widely used process for the biological treatment of domestic and industrial wastewaters. Wastewater treatment plants using the activated sludge process are in widely used in developed and developing countries. The activated sludge model number 1 (ASM \#1) is an internationally accepted standard for activated sludge modelling. It describes nitrogen and chemical oxygen demand within suspended-growth treatment processes, including mechanisms for nitrification and denitrification.
\end{abstract}

We analysed the biological treatment of a wastewater when a cascade of five reactors were used. Operating conditions were investigated in which the first reactor was not aerated. The second reactor could be either be aerated or not aerated. The remaining reactors were aerated. The process configuration included one settling tank and one recycle step. Both of these were placed after the final reactor in the cascade.

We used the hydraulic retention time (HRT) as the bifurcation parameter and investigate how the total nitrogen concentration in the effluent $\left(\mathrm{TN}_{e}\right)$ stream depended upon the operation of the recycle step and the state of aeration in the second reactor. When the reactor configuration included an ideal settling tank, the total nitrogen concentration is defined as

$$
\mathrm{TN}_{e}=S_{\mathrm{NO}}+S_{\mathrm{NH}}+S_{\mathrm{ND}},
$$

where the state variables on the right hand side are the concentration of soluble nitrate and nitrite nitrogen $\left(S_{\mathrm{NO}}\right)$, soluble ammonium nitrogen $\left(S_{\mathrm{NH}}\right)$, and soluble biodegradable organic $\left(S_{\mathrm{ND}}\right)$ respectively.

We explored a number of different aspects of a five reactor cascade.

1. We compared the performance of reactor configurations up to five reactors, with the first reactor not aerated. The qualitative behaviour was the same, with three regimes of HRT: for low HRT, the system was in a washout state, where the process failed, then a transition, or bifurcation, when the heterotrophic biomass species were present, and finally another transition, when both the heterotrophic and autotrophic biomass species were present. The final transition occurred at lower HRT as the number of reactors in the configuration was increased. There was also an improvement in the performance.

2. We explored the effect of varying the HRT in each reactor and compared the performance with an equal HRT configuration. For the most of total HRT values, the equal HRT configuration offered close to the best performance. For a total HRT near the transition where the heterotrophic and autotrophic biomass species were present, the equal HRT configuration offered close to the worst performance.

3. We explored the effect the configuration with a recycling step, compared to a configuration where there was no recycling step. We found that the configuration with a recycling step outperformed one without. However, the performance improvement occurred up to critical recycling rate. For higher recycling ratios, the performance deteriorated.

4. Finally, we explored the effect of either aerating the first and second reactors, while aerating the other three reactors, not aerating the first reactor and aerating the second or not aerating the first and second reactors. We found that it was better to not aerate the first reactor and aerate the remaining reactors.

Keywords: Activated sludge, modelling, recycling, stirred tank, wastewater 


\section{INTRODUCTION}

The activated sludge process is the most commonly used aerobic process for the biological treatment of both domestic and industrial wastewaters [Wei et al., 2003]. As such, most wastewater treatment plants contain a unit employing the activated sludge process. The key to this process is the presence of highly concentrated micro-organisms, typically 2-4 g/L, present in the form of flocs, which grow though consuming organic pollutants. The mixture of flocs and particulate matter is known, for historical reasons, as "activated sludge". Without sufficient quantities of sludge, i.e. micro-organisms, the process cannot work.

The simplest reactor configuration for the activated sludge process entails the use of two steps: a series of biological reactors and a settling tank (or clarifier). In the former the pollutants are degraded by microorganisms (the active agent that puts the 'activated' into 'activated sludge'). Inside each of the bioreactors the microorganisms flocculate to form settleable solids. These solids are removed from the effluent stream by sedimentation in a settling tank and then returned to the first reactor in a more concentrated culture. The use of a clarifier ensures the presence of a culture of highly concentrated micro-organisms and is one of the keys to the success of the activated sludge process.

Modelling has become an important tool to develop an understanding of the processes that govern the behaviour of the activated sludge process. A widely used model that describes the biological processes occurring in the activated sludge process is the activated sludge model \#1 (ASM \#1) [Henze et al., 1987|. This consists of a biochemical model for the particulates and soluble materials and a sub-model describing nitrifying processes.

We investigate the steady-state behaviour of the ASM \#1 as a function of the hydraulic retention time. (The hydraulic retention time is defined as the volume of the reactor divided by the feed flow rate. It represents the average amount of time that a soluble compound remains in the reactor.) From the steady-state values of the state variables we can calculate the corresponding steady-state values of various quantities associated with the operation of the activated sludge process, such as the chemical oxygen demand, the total suspended solids, total nitrogen and total inorganic nitrogen. We will measure the performance of the system using the total nitrogen.

\section{MATERIALS AND METHODS}

We implement the Activated Sludge Model number 1 (ASM \#1) [Henze et al., 1987| in a five-reactor cascade containing settling tank and a recycle step. The settling tank is positioned after final reactor and the recycling step is from the final reactor to the first reactor (part of the exit stream from the settling tank is discharged as waste). A schematic of the reactor configuration is shown in Figure 1. To model the settling tank we used

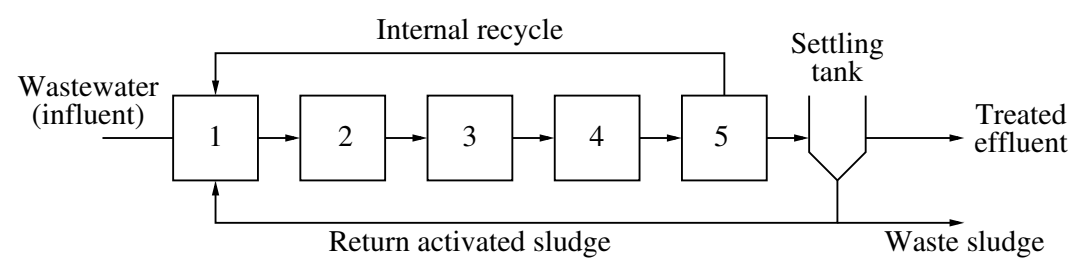

Figure 1. A schematic of the bioreactor configuration with two recycling steps and a settling tank.

the ideal settling tank model. This characterises the settling tank by three parameters. These are the recycle ratio $(R)$, the concentration factor of paritculates $(\mathcal{C})$, and the fraction of the non-effluent stream leaving the settling tank that is discharged to waste $(w)$. The recycle step is characterised by one parameter: a recycle ratio $\left(R_{5 \rightarrow 1}\right)$.

See Henze et al. [1987] for a detailed description of the ASM \#1 and to Nelson et al. [2019] for a full nomenclature and a complete listing of parameter values. The latter also provides the differential equations for a single reactor connected to a settling tank.

\subsection{State variables}

The ASM \#1 incorporates differential equations for six soluble and six particulate components. The six soluble components are: inert soluble organic material, readily biodegradable soluble substrate, soluble oxygen, 
soluble nitrate \& nitrite nitrogen (one component), soluble ammonium $\left(\mathrm{NH}_{4}^{+}\right.$and $\mathrm{NH}$ ) nitrogen, and soluble biodegradable organic nitrogen. The particulate components are: particulate inert organic matter, slowly biodegradable particulate substrate, active heterotrophic particulate biomass, active autotrophic particulate biomass, non-biodegradable particulate products arising from biomass decay, and particulate biodegradable organic nitrogen.

\subsection{Formulation of reaction rates}

The ASM \#1 includes eight fundamental processes which describe nitrogen and chemical oxygen demand within suspended-growth treatment processes, These are: (1) aerobic growth of heterotrophic biomass, (2) anoxic growth of heterotrophic biomass, (3) aerobic growth of autotrophic biomass, (4) decay of heterotrophic biomass, (5) decay of autotrophic biomass, (6) ammonification of soluble organic nitrogen, (7) hydrolysis of entrapped particulate organic matter and (8) hydrolysis of entrapped organic nitrogen. These processes includes mechanisms for nitrification and denitrification

In writing the equations we use the symbol $S_{O, k}$ to denote the concentration of soluble oxygen in the $k$ th reactor $(k=1 \ldots 5)$ and the symbol $S_{j, k}(j=1 \ldots 5)$ to denote the concentration of the five other soluble components in the $k$ th reactor. We denote the concentration of the six particulate components in the $k$ th reactor by $X_{l, k}(l=1 \ldots 6)$. The rate functions are specified in a column vector. The $i$ th row of this vector is written $\mathbf{f}_{i}\left(\mathbf{S}_{k}, \mathbf{X}_{k}\right)$. The $i$ th reaction rate in reactor $k$ depends upon the the corresponding concentration of the soluble and particulate components. In this formulation the variable $\mathbf{S}_{k}$ includes all six of the soluble components. The reaction rate expression may contain as few as one of the twelve state variables. (Two of the state variables appear in no reaction rate expressions.)

For each of the state variables there is a stoichiometric column vector $\alpha_{i, j}$. The second index $(j=1,2, \ldots 12)$ identifies the state variables being considered. The first index $(i=1,2, \ldots 8)$ indicates the process being considered (as numbered above). If the entry $\alpha_{i, j}$ is positive, then component $j$ is a product in reaction $i$. If the entry $\alpha_{i, j}$ is negative, then component $j$ is a reactant in reaction $i$. If the entry $\alpha_{i, j}$ is zero, then component $j$ is neither a product nor a reactant in reaction $i$.

\subsection{Characterisation of the activated sludge process}

The concentrations of each of the twelve state variables are determined in each reactor. Various linear combinations of the state variables are of particular interest. These combinations define different process variables, such as chemical oxygen demand (COD), total suspended solids (TSS), total nitrogen (TN), and total inorganic nitrogen. These variables may be evaluated in the influent stream, in each of the reactors, in the sludge waste stream, and in the effluent stream. We consider the total nitrogen concentration in the effluent stream.

\section{MODEL EQUATIONS}

\subsection{Model equations in the first reactor}

Rate of change of soluble components (excluding oxygen) in the first reactor $(j=1 \ldots 5)$

$$
\frac{\mathrm{d} S_{j, 1}}{\mathrm{~d} t}=\frac{1}{\tau_{1}}\left(S_{j, \text { in }}-S_{j, 1}\right)+\frac{R}{\tau_{1}}\left(S_{j, 5}-S_{j, 1}\right)+\frac{R_{5 \rightarrow 1}}{\tau_{1}}\left(S_{j, 5}-S_{j, 1}\right)+\sum_{i=1}^{8} \alpha_{i, j} \cdot \mathbf{f}_{i}\left(\mathbf{S}_{1}, \mathbf{X}_{1}\right) .
$$

Rate of change of soluble oxygen in the first reactor

$$
\begin{aligned}
\frac{\mathrm{d} S_{\mathrm{O}, 1}}{\mathrm{~d} t}= & \frac{1}{\tau_{1}}\left(S_{O, \text { in }}-S_{\mathrm{O}, 1}\right)+\frac{R}{\tau_{1}}\left(S_{\mathrm{O}, 5}-S_{\mathrm{O}, 1}\right)+\frac{R_{5 \rightarrow 1}}{\tau_{1}}\left(S_{\mathrm{O}, 5}-S_{\mathrm{O}, 1}\right)+K_{\mathrm{L}, \mathrm{a}, 1}\left(S_{\mathrm{O}, \max }-S_{\mathrm{O}, 1}\right) \\
& +\sum_{i=1}^{8} \alpha_{i, O} \cdot \mathbf{f}_{i}\left(\mathbf{S}_{1}, \mathbf{X}_{1}\right) .
\end{aligned}
$$

Rate of change of particulate components in the first reactor $(l=1 \ldots 6)$

$$
\frac{\mathrm{d} X_{l, 1}}{\mathrm{~d} t}=\frac{1}{\tau_{1}}\left(X_{l, \text { in }}-X_{l, 1}\right)+\frac{R}{\tau_{1}}\left(\mathcal{C} X_{l, 5}-X_{l, 1}\right)+\frac{R_{5 \rightarrow 1}}{\tau_{1}}\left(X_{l, 5}-X_{l, 1}\right)+\sum_{i=1}^{8} \alpha_{i, l} \cdot \mathbf{f}_{i}\left(\mathbf{S}_{1}, \mathbf{X}_{1}\right) .
$$


In these equations the parameters are: $\mathcal{C}$, the concentration factor for particulates in the settling tank; $K_{\mathrm{L}, \mathrm{a}, 1}$, the oxygen transfer coefficient in the first reactor; $R$, the recycle ratio of the settling tank; $R_{5 \rightarrow 1}$, the recycle ratio of the recycle step; $S_{j \text {,in }}$, the concentration of soluble component $j$ in the feed; $S_{O \text {,in }}$, the concentration of soluble oxygen in the feed; $S_{O, \max }$, the maximum concentration of soluble oxygen; $X_{l, \text { in }}$ is the concentration of the particulate components $l$ in the feed, and $\tau_{1}$, the hydraulic retention time in the first reactor.

\subsection{Model equations in reactors two to five}

Rate of change of soluble components (excluding oxygen) in the $k$ th reactor $(j=1 \ldots 5)$

$$
\frac{\mathrm{d} S_{j, k}}{\mathrm{~d} t}=\frac{1+R+R_{5 \rightarrow 1}}{\tau_{k}}\left(S_{j, k-1}-S_{j, k}\right)+\sum_{i=1}^{8} \alpha_{i, j} \cdot \mathbf{f}_{i}\left(\mathbf{S}_{k}, \mathbf{X}_{k}\right)
$$

Rate of change of soluble oxygen in the $k$ th reactor

$$
\begin{aligned}
\frac{\mathrm{d} S_{\mathrm{O}, k}}{\mathrm{~d} t}= & \frac{1+R+R_{5 \rightarrow 1}}{\tau_{1}}\left(S_{O, k-1}-S_{\mathrm{O}, k}\right)+K_{\mathrm{L}, \mathrm{a}, k}\left(S_{\mathrm{O}, \max }-S_{\mathrm{O}, k}\right) \\
& +\sum_{i=1}^{8} \alpha_{i, O} \cdot \mathbf{f}_{i}\left(\mathbf{S}_{k}, \mathbf{X}_{k}\right) .
\end{aligned}
$$

Rate of change of particulate components in the $k$ th reactor $(l=1 \ldots 6)$

$$
\frac{\mathrm{d} X_{l, k}}{\mathrm{~d} t}=\frac{1+R+R_{5 \rightarrow 1}}{\tau_{k}}\left(X_{l, k-1}-X_{l, k}\right)+\sum_{i=1}^{8} \alpha_{i, l} \cdot \mathbf{f}_{i}\left(\mathbf{S}_{k}, \mathbf{X}_{k}\right)
$$

In these equations the parameters are: $K_{\mathrm{L}, \mathrm{a}, k}$, the oxygen transfer coefficient in the $k$ th reactor; and $\tau_{k}$, the hydraulic retention time in the $k$ th reactor. The details of the reaction rates $\mathbf{f}$ are given in Nelson et al. [2019].

\section{RESULTS AND DISCUSSION}

The steady state behaviour of the ODE system are analysed. From a starting concentration of each of the state variables, the state variable will evolve to one of a number of solution paths. In general, there will be only one possible solution path for a given set of parameters. As the system parameters change, the solution path followed can change if the solution path becomes unstable when one of the eigenvalues of the linearised system becomes positive.

\subsection{Total nitrogen in the effluent stream: the effect of deploying more reactors}

Figure 2 shows the total nitrogen in the effluent stream as the number of identical reactors is increased. For small hydraulic retention times (HRT $<0.053$ days), the system evolves to the washout state, where the autotrophic and heterotrophic biomass have zero concentration (in real world situations, there would a very small amount present). When the autotrophic and heterotrophic biomass have zero concentration, all reactor rates $\mathbf{f}$ are zero and the activated sludge process fails.

As the hydraulic residence time (HRT) is increased, the washout solution becomes unstable. There is a sudden decrease in the total nitrogen as another solution path is followed in which heterotrophic biomass is present. A second bifurcation occurs as the HRT is increased further. Following this the total nitrogen initially decreases rapidly, with a much slower decrease at larger residence times. In this state, both biomass are present. As the number of reactors in the system increases, this second transcritical bifurcation occurs at lower HRTs.

\subsection{Total nitrogen in the effluent stream: the effect of optimising the reactor size}

In the simulations shown in Figure 2 it is assumed that each reactor in the cascade is of equal size. It is reasonable to ask whether an improvement in the performance of a cascade can be obtained by varying the relative 'size' of the reactors in the cascade. Rather than varying the volume of each reactor we conceptualise this as varying the flow rate through each reactor. Mathematically, this can be achieved by adjusting the HRT in each reactor. 


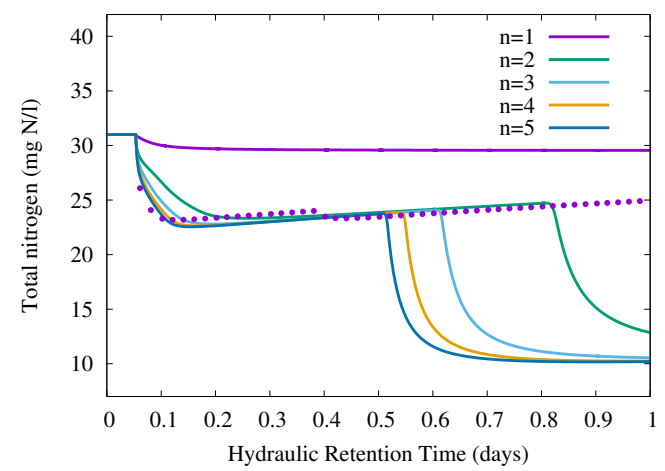

Figure 2. Total nitrogen leaving the reactor cascade as a function of the hydraulic retention time. The solid lines indicate reactor configurations in which the first reactor is nor aerated. The dotted line represents an aerated one-reactor configuration. The value of the oxygen transfer coefficient in the aerated reactors is $K_{L, a, k}$ $=108$ day $^{-1}$ [Fikar et al., 2005]. The recycle ratios through the settling tank and recycle step are $R=0.45$ and $R_{k \rightarrow 1}=1$, respectively.

We investigate representative total HRT (summing the HRT in each reactor) of: four, eight, twelve, sixteen, twenty and twenty-four hours. We consider reactor configurations where the first reactor is not aerated, but the remaining reactors are aerated. For example, we consider (in $5 \%$ increments) all five-reactor configurations such that the sum of the HRT in each reactor is four hours. For each total HRT, we can calculate the optimal total nitrogen. The results are shown in Table 1 and Figure 3. The vertical lines in Figure 3 represent the range of total nitrogen in the effluent for each of the possible five reactor configurations for a given total HRT. These results suggest that for most residence times there is little advantage in optimising the reactor configuration. However, for residence times near the second transcritical bifurcation the equal reactor configuration delivers a poor performance compared against some of the other configurations.

Table 1. Comparison of the equal reactor configuration and the optimal reactor configuration. It is assumed that only the first reactor is not aerated. The values of the recycling ratios for the recycling step and the settling tank are the same as those in Figure 2.

\begin{tabular}{|c|c|c|c|}
\hline $\begin{array}{c}\text { Total residence time } \\
\text { (hours) }\end{array}$ & $\begin{array}{c}\text { Equal reactor } \\
\text { performance }(\mathrm{mg} \text { N/l) }\end{array}$ & $\begin{array}{c}\text { Optimal configuration } \\
\text { performance }(\mathrm{mg} / \mathrm{l})\end{array}$ & $\begin{array}{c}\text { Improvement } \\
(\%)\end{array}$ \\
\hline 4 & 22.56 & 22.53 & 0.1088 \\
8 & 23.13 & 23.12 & 0.0727 \\
12 & 23.73 & 13.60 & 42.7129 \\
16 & 10.64 & 10.50 & 1.2725 \\
20 & 10.19 & 10.14 & 0.3998 \\
24 & 10.20 & 10.14 & 0.5924 \\
\hline
\end{tabular}

\subsection{Total nitrogen in the effluent stream: the effect of the recycle rate through the recycling step}

Figure 4 shows how the nitrogen concentration in the effluent stream depends upon the operation of the recycle step. As the recycling rate increases the HRT at the second bifurcation decreases. For a fixed value of the HRT the nitrogen concentration decreases as the value of $R_{5 \rightarrow 1}$ increases from zero to two. However, for higher values of $R_{5 \rightarrow 1}$ the variation is more subtle: the optimal value for $R_{5 \rightarrow 1}$ depends upon the value of the HRT.

In Figure 4 we have included a target total nitrogen of $10 \mathrm{mg} \mathrm{N} / \mathrm{l}$. The performance of configurations with recycling ratios $R_{5 \rightarrow 1}=2$ and $R_{5 \rightarrow 1}=3$ both cross this line at around 0.6 days. 


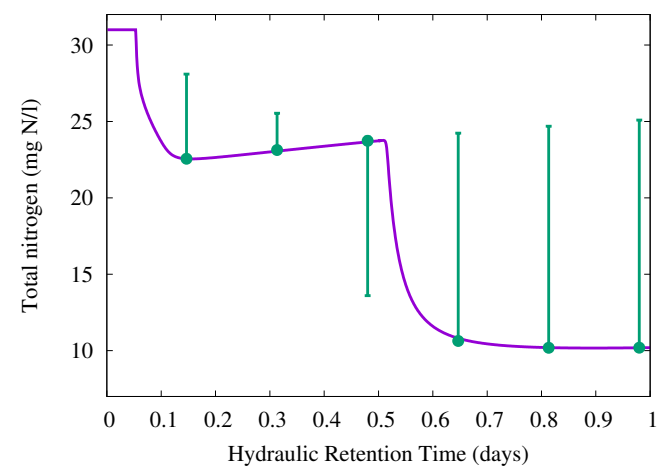

Figure 3. Comparison of the equal reactor profile (purple line) with all possible five-reactor configurations (green line). The values of the recycling ratios for the recycling step and settling tank are those in Figure 2.

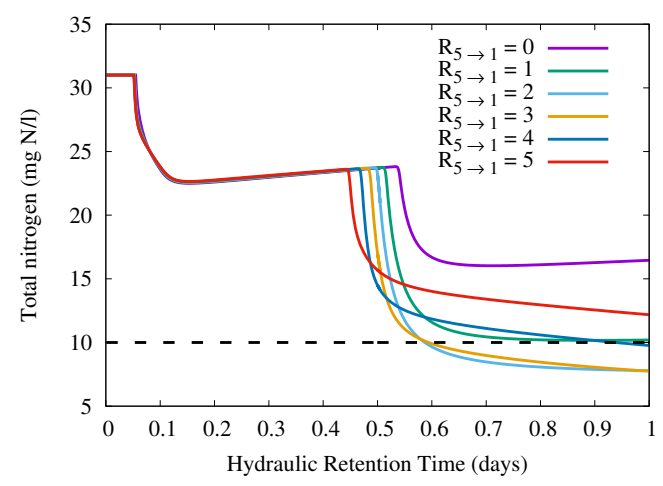

Figure 4. Total nitrogen leaving the reactor cascade as a function of the hydraulic retention time and the recycle rate through the recycling step $\left(R_{5 \rightarrow 1}\right)$. The dashed line represents the target of $10 \mathrm{mg}$ for the total Nitrogen content. The recycle rate through the settling step is $R=0.45$.

\subsection{Total nitrogen in the effluent stream: the effect of not aerating all reactors}

Previously we assumed that only the first reactor was not aerated. We then investigated the effect of not aerating the second reactor. A comparison of three configurations (all reactors a erated; first reactor not ae rated; first and second reactors not aerated) is shown in Figure 5. We see that the onset of the second bifurcation is earlier when all reactors are aerated, but when the first reactor is not aerated the performance improves beyond the bifurcation point. Not aerating the second reactor does not improve performance. Previous results suggest that this may not hold for higher values of the recycling rate [Nelson and Hai, 2019] and is an area of future work.

\section{MODEL LIMITATIONS AND FUTURE WORK}

The model we have investigated in this paper is an extension of the model studied by Nelson and Hai [2019], where the number of reactors is extended from four to five. Depending on the wastewater and the treatment level required, three, four or fives reactor configurations were us ed. For three reactors, consisting of an anaerobic reactor (a reactor where there is no molecular oxygen or nitrite), an anoxic reactor (a reactor without aeration, but which receives enriched nitrite from an aerated reactor) and an aerobic reactor, we expect carbonaceous organics removal, denitrification and significant phosphorous removal. For four reactors, consisting of two anoxic reactors and two aerobic reactors, there is no phosphorous removal as this requires an anaerobic reactor. For five reactors, consisting of a n a naerobic reactor, two a noxic reactors and two a erobic reactors, we expect carbonaceous organics removal, denitrification and phosphorous $r$ emoval. The five reactor system should be more stable than the three reactor configuration because of the double anoxic and double aerobic reactors. This behaviour can not be investigated using the ASM \#1 as phosphorous is not included in the model. In the future we hope to explore one of the variants of the Activated Sludge Model number 2 (ASM \# 


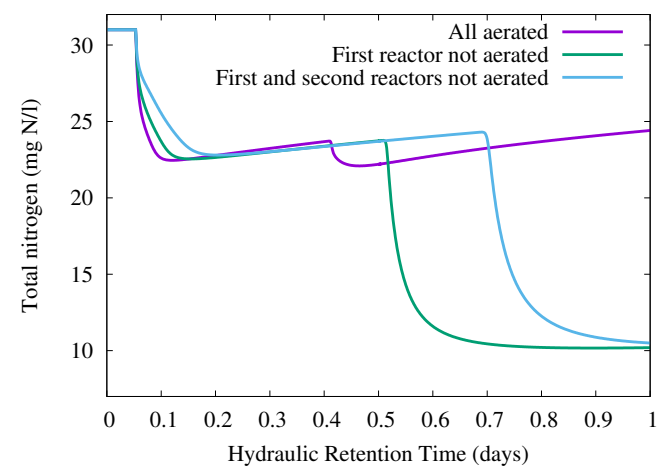

Figure 5: Total nitrogen leaving the reactor cascade $(\mathrm{mg} \mathrm{N} / \mathrm{l})$ as a function of the hydraulic retention time (days) for different aeration configurations. The values of the recycling ratios for the recycling and settling tank are the same as those in Figure 2

2) [Gujer et al., 1995|, as this will include phosphorous.

\section{CONCLUSIONS}

We investigated the ASM \#1 model for the treatment of wastewater by the activated sludge process. The process configuration is a five reactor cascade with a settling tank and a recycle step after the final reactor. We primarily investigated the effect of varying the value of the recycle ratio of the recycling step under the condition that the reactors are of equal size and that the first reactor is not aerated. We characterised the performance of the treatment from the change in nitrogen concentration in the effluent streams.

The performance of the system improved as the number of reactors increases, though this improvement reduces as the HRT increases. The HRT where a second transcritical bifurcation occurs and which corresponds to significantly decreased nitrogen concentrations, decreases as the number of reactors increases.

We explored the effect of varying the relative sizes of the reactors. For most values of the HRT the case of equal reactors was close to the optimal configuration. However, at HRTs near the second transcritical bifurcation the equal reactor configuration was close to the worst configuration.

Finally, we considered the effect of aeration on the performance. Three configurations were considered: all reactors aerated; the first reactor not aerated; the first and second reactors not aerated. Removing aeration from the second reactor did not improve the performance of the system.

\section{REFERENCES}

M. Fikar, B. Chachuat, and M.A. Latifi. Optimal operation of alternating activated sludge processes. Control Engineering Practice, 13:853-861, 2005. doi: https://doi:10.1016/j.conengprac.2004.10.003.

W. Gujer, M. Henze, T. Mino, T. Matsuo, M.C. Wentzel, and G.v.R. Marais. The activated sludge model no. 2: Biological phosphorus removal. Water Science and Technology, 31(2):1-11, 1995. ISSN 0273-1223. doi: https://doi.org/10.1016/0273-1223(95)00175-M. URL https://www. sciencedirect.com/ science/article/pii/027312239500175M.

M. Henze, C.P. Leslie Grady, W. Gujer, G.V.R. Maris, and T. Matsuo. Activated sludge process model no 1. Scientific and Technical Report 1, IAWQ, London, UK, 1987.

M.I. Nelson and F.I. Hai. Nitrogen removal in a cascade of four reactors employing the activated sludge process. In S. El Sawah, editor, MODSIM2019, Canberra, 01 December 2019, 2019. doi: https://dx.doi. org/10.36334/modsim.2019.A5.nelson.

M.I. Nelson, H.S. Sidhu, S. Watt, and F.I. Hai. Performance analysis of the activated sludge model (number 1). Food and Bioproducts Processing, 116:41-53, 2019. URL http://www . sciencedirect.com/ science/article/pii/s0960308519302871

Y. Wei, Renze T. Van Houten, A. R. Borger, D. H. Eikelboom, and Y. Fan. Minimization of excess sludge production for biological wastewater treatment. Water Research, 37(18):4453-4467, 2003. ISSN 00431354. doi: https://doi.org/10.1016/S0043-1354(03)00441-X. URL https : / / www . sciencedirect.

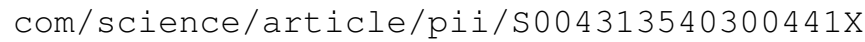

